\title{
Characterising the Clinical Spectrum, Diagnosis and Outcomes in Secondary Stress Cardiomyopathy
}

\author{
Puneet Gupta and Anand Chockalingam \\ Division of Cardiovascular Medicine, University of Missouri, Columbia, MO, USA
}

\begin{abstract}
ntroduction: Available literature on takotsubo cardiomyopathy excludes critically ill patients due to challenges in angiographic confirmation. Secondary stress cardiomyopathy (SSC) occurs in patients already hospitalised for other critical illnesses. Diagnosis of sSC is challenging, while clinical presentation and outcomes are significantly different from primary stress cardiomyopathy. Our aim was to better characterise the clinical picture of SSC. Methods: The diagnosis of SSC was confirmed based on characteristic clinical and morphological features, applying our diagnostic algorithm suited for critically ill patients. We were able to characterise these sSC patients and differentiate their presentation from takotsubo registry population. Data on selected patients was extracted manually on Microsoft Excel worksheets with relevant patient demographics, presenting features and outcomes. Results: We developed a profile of sSC based on 18 consecutive confirmed cases diagnosed at our university hospital between April 2016 and September 2018. sSC differed from takotsubo cardiomyopathy in several key clinical aspects - younger people may develop sSC (range 21-86 years) and men were more frequently affected in comparison to takotsubo cardiomyopathy (29\%). Dyspnoea was noted in $22 \%$ of our patients and angina was rare. Apical ballooning occurred in only $33 \%$ of the patients, while mid (39\%) and basal left ventricular (11\%) variants accounted for half of the patients. Mortality was much higher (28\%) due to underlying medical comorbidities. Conclusions: Our series illustrates significant clinical and morphologic differences in the presentation of sSC. Shifting the emphasis to serial echocardiography would reduce the need for invasive catheterisation and downstream comorbidity in critical care settings.
\end{abstract}

\section{Keywords}

Stress cardiomyopathy, secondary stress cardiomyopathy, takotsubo, critically ill, echocardiography, diagnosis

Disclosure: Puneet Gupta and Anand Chockalingam have nothing to declare in relation to this article.

Review Process: Double-blind peer review.

Compliance with Ethics: All procedures were followed in accordance with the responsible committee on human experimentation and with the Helsinki Declaration of 1975 and subsequent revisions. This study was retrospective chart review of existing observational data. Authorship: The named authors meet the International Committee of Medical Journal Editors (ICMJE) criteria for authorship of this manuscript, take responsibility for the integrity of the work as a whole, and have given final approval for the version to be published.

Received: 15 August 2019

Accepted: 18 October 2019

Citation: Heart International. 2019;13(2):26-30

Corresponding Author: Anand Chockalingam, Division of Cardiovascular Medicine, University of Missouri - Columbia, Five Hospital

Drive, CE306, Columbia, MO 65212, USA.

E: chockalingama@health.missouri.edu

Support: No funding was received in

the publication of this article.
First described in Japan in 1990, takotsubo or stress cardiomyopathy (SC), has become one of most commonly described forms of cardiomyopathy in the literature over the past two decades. Typically, patients with SC present with angina or other acute cardiac symptoms in the setting of significant physical or mental stressors and transient myocardial dysfunction which resolves within a few weeks. Coronary angiography reveals no acute lesions and these patients tend to have a relatively better prognosis compared to acute coronary syndromes. ${ }^{2-3}$ Several diagnostic criteria pertaining to this form of SC (referred interchangeably as primary stress cardiomyopathy [pSC] for the remaining portion of this article) have been published over the past decade..$^{2-5}$ Over the past few years, a subgroup of these patients have been increasingly recognised, particularly in the critically ill population. These patients present for medical attention for a variety of critical illnesses and tend to develop features of SC during the course of their hospital stay. These critically ill patients developing cardiac issues differ from the typically described patients with SC in many aspects. Very little systematic data is available to characterise the true scope and outcomes in this form of SC. First proposed in 2016, the term secondary SC (SSC) describes SC developing during the hospitalisation of critically ill patients. ${ }^{2}$ This clinical subtype is much more heterogeneous and was defined in a recent review as occurring in 'patients already in the healthcare setting, during evaluation or treatment of another critical illness and often present insidiously in a wide range of clinical and surgery settings. ${ }^{6}$

The International Takotsubo Registry (InterTAK) was the first large-scale effort to characterise the clinical features and outcomes of SC. ${ }^{3}$ The 2008 revised Mayo criteria, ${ }^{5}$ which were the basis for inclusion in the registry, require angiographic exclusion of obstructive coronary lesions. Although the recently published InterTAK registry diagnostic criteria and their scoring system are increasingly used tools in the diagnosis of $\mathrm{SC}_{1}^{7}$ they do not appear to truly characterise the clinical spectrum and prognosis of patients with SSC, since critically ill patients were often excluded in the registry due to lack of angiographic confirmation of SSC. An analysis of takotsubo cardiomyopathy patients from the Registry on Takotsubo Syndrome (RETAKO) group in 2016 classified all patients with physical triggers as SSC, but only those patients who fulfilled Mayo criteria were included. ${ }^{8}$ Since angiographic exclusion of obstructive coronary artery disease (CAD) was required, the study may have excluded many patients with possible SC, particularly the critically ill patients with SSC. This study did suggest worse short- and long-term prognosis in such patients, although the analysis may not have included the entire spectrum of patients with SSC. 


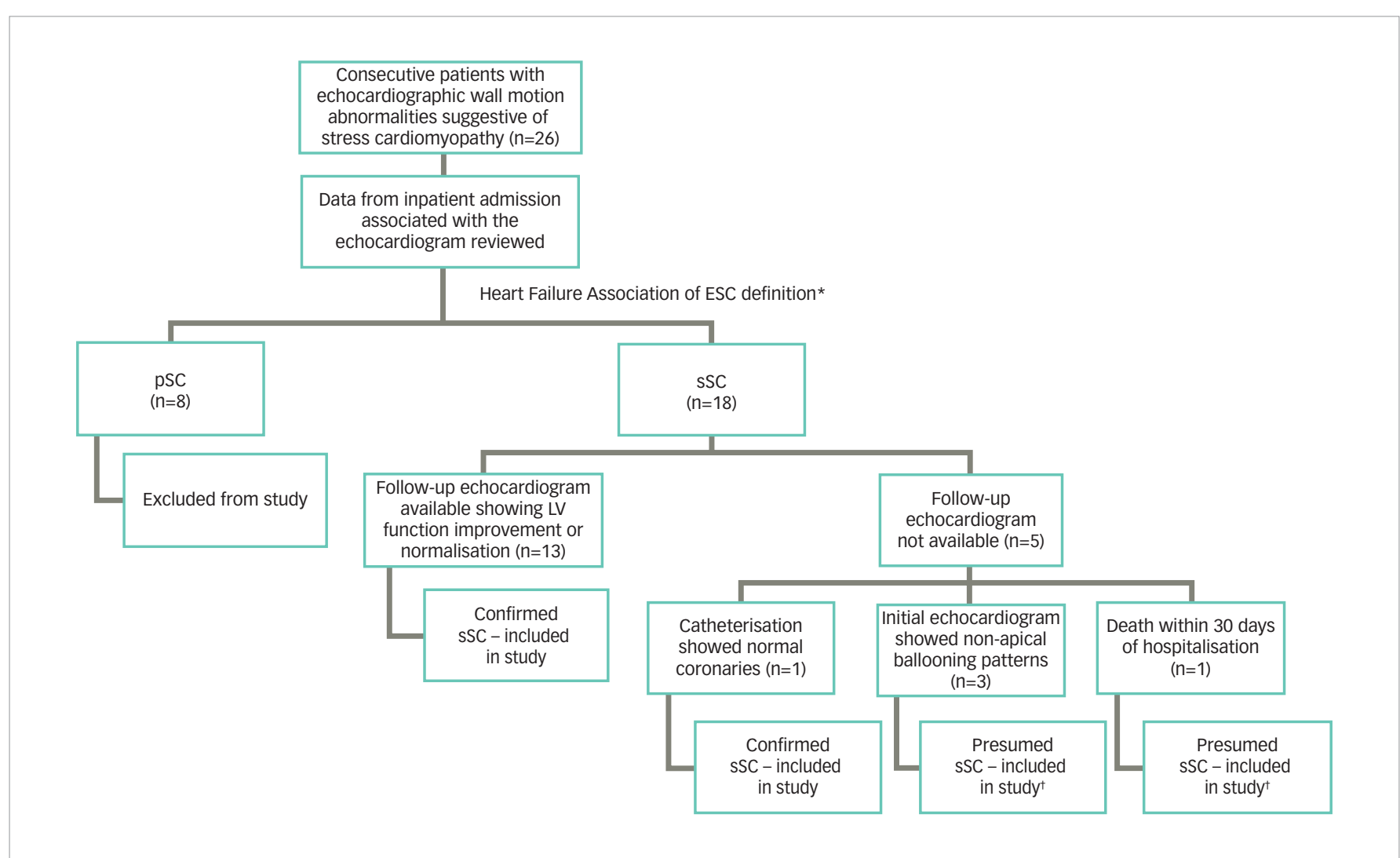

*As per the definitions of the Heart Failure Association of the ESC, those patients in whom acute cardiac symptoms were the primary reason for seeking medical care were diagnosed pSC and those cases in whom hospitalisation resulted for other medical, surgical, obstetric, anaesthetic or psychiatric conditions, were diagnosed with sSC. ${ }^{t}$ pSC was already excluded based on definition.

ESC = European Society of Cardiology; LV = left ventricular; $p S C=$ primary stress cardiomyopathy; SSC = secondary stress cardiomyopathy.

Due to these challenges in the available studies, systematic serial data defining the characteristics of patients with SSC and their prognosis, are largely lacking. This case series was our attempt to define the clinical and morphologic characteristics of patients with SSC.

Our group has been interested in the non-invasive diagnosis of SC for over a decade. Our echocardiography-based algorithm to diagnose sSC without requiring invasive catheterisation has been published elsewhere. ${ }^{9}$ In our 2017 review, we defined SSC as acute cardiac dysfunction developing during the course of hospitalisation for critical medical, surgical or neurological illness. ${ }^{10}$ The present single-centre experience of consecutive sSC cases was collected to gain a better understanding of its true clinical spectrum in a university hospital setting.

\section{Materials and methods}

For the purpose of this study, 26 consecutive patients with characteristic echocardiographic patterns of regional wall motion abnormalities noted by a single cardiologist, were included. The five patterns of echocardiographic patterns of regional wall motion abnormalities considered, were: apical ballooning/hypokinesis associated with basal hyperkinesis, isolated mid-ventricular hypokinesis, isolated basal hypokinesis, focal hypokinesis, and biventricular dysfunction/ global hypokinesia. Patients with wall motion abnormalities thought to represent a single epicardial coronary distribution were excluded. since a significant proportion of these patients could not be taken to cardiac catheterisation due to underlying comorbidities, angiographic exclusion of CAD was not required to diagnose SSC. If a repeat echocardiogram obtained up to a few months later showed recovery of these wall motion abnormalities, then the diagnosis of sSC was made non-invasively. If a follow-up echocardiogram was not available, due to mortality within 30 days of hospitalisation, those patients were still included in the study.

A detailed algorithm representing the study design is presented in Figure 1. Data were collected retrospectively from the hospital's electronic medical record system on the identified patients. We specifically collected demographic information, specifics of presentation, electrocardiograms, possible triggers, echocardiographic patterns of wall motion abnormalities and outcome data which primarily included in-hospital and 30-day mortality. The clinical presentation of the study patients was then categorised as being consistent with SSC or with pSC, based on the definitions recently published by the Heart Failure Association of the European Society of Cardiology. ${ }^{2}$ As per these definitions, those patients in whom acute cardiac symptoms were the primary reason for seeking medical care were diagnosed pSC and those cases in whom hospitalisation resulted for other medical, surgical, obstetric, anaesthetic or psychiatric conditions, were diagnosed with sSC. ${ }^{2}$ In general, patients who did not have follow-up echocardiographic data were excluded, with the exception of patients who died in hospital or within 30 days of discharge, preventing a follow-up echocardiogram to be obtained. None of these patients without follow-up echocardiograms had pSC, as this group was separated earlier based on the definitions of the Heart Failure Association of the European Society of Cardiology (acute cardiac symptoms were not the primary reason for seeking medical care; Figure 1). Other exceptions included the non-apical ballooning variants (diagnosis of SSC was presumed in this situation because PSC was 
Table 1: Baseline characteristics of patients with secondary stress cardiomyopathy

\begin{tabular}{|l|l|}
\hline Average age (range) & 61 years (21-86) \\
\hline Diabetes mellitus & $6(33)$ \\
\hline Hypertension & $9(50)$ \\
\hline Prior CAD & $2(11)$ \\
\hline Female & $14(78)$ \\
\hline Depression & $4(22)$ \\
\hline Hyperlipidaemia & $9(50)$ \\
\hline COPD & $2(11)$ \\
\hline Shock & $8(44)$ \\
\hline ICU admission & $10(56)$ \\
\hline
\end{tabular}

Data are presented as $n(\%)$ unless otherwise stated. $n=18$.

$C A D=$ coronary artery disease $; C O P D=$ chronic obstructive pulmonary disease, ICU = intensive care unit.

Table 2: Chief complaints on presentation in patients with secondary stress cardiomyopathy

\begin{tabular}{|l|l|}
\hline Angina & $0(0)$ \\
\hline Dyspnoea & $4(22)$ \\
\hline Altered mental status & $2(11)$ \\
\hline Unresponsiveness/found down & $4(22)$ \\
\hline Other* & $8(44)$ \\
\hline
\end{tabular}

* Other complaints include nausea, vomiting, headache, weakness, fall, facial droop and vertigo.

Data are presented as $n(\%)$.

already excluded based on definition detailed above) as these patterns do not correspond to a single epicardial coronary artery distribution. One patient underwent catheterisation excluding CAD. With the application of this algorithm, all 18 patients with sSC were included in the study.

\section{Results}

A total of 26 consecutive patients were initially included in this study; 18 patients were diagnosed with SSC and 8 with pSC. All patients had critical illness on presentation to the hospital. The baseline characteristics are presented in Table 1. The average age of patients in our series was 61 years, ranging from 21 to 86 years. This sSC group had a significantly higher proportion of younger patients compared to pSC in the INTERTAK registry. Women were affected more frequently than men as traditionally quoted for patients with SC, although the proportion of men was higher. Key triggers for sSC in our group were cardiac arrest, ventricular tachycardia, ventricular fibrillation, pulseless electrical activity, respiratory failure, attempted suicide by hanging, intracranial haemorrhage, stroke, sepsis, surgery and motor vehicle accident. None of the patients in our series had previously documented left ventricular (LV) dysfunction.

In contrast to patients with pSC, the majority of patients in our series did not seek medical attention due to angina (Table 2). Dyspnoea was noted in some patients (22\%) on presentation. Other patients presented with neurological, psychiatric, or non-specific symptoms. Electrocardiograms (ECGS) demonstrated a variety of changes including ST segment elevation (not meeting criteria for ST segment elevation myocardial infarction) or depression, QRS prolongation, atrial or ventricular tachy-arrhythmias. We also collected serial troponin T levels obtained during hospitalisation for all patients in the series (Table 3). In general, serum troponin level elevations were modest $(<1 \mathrm{ng} / \mathrm{mL}$ ) in most patients,
Table 3: Trend of troponin $\mathrm{T}(\mathrm{ng} / \mathrm{mL})$ in all patients with secondary stress cardiomyopathy during hospitalisation

\begin{tabular}{|c|c|c|c|}
\hline Patient & First TnT level & Second TnT level & Third TnT level \\
\hline 1 & 1.11 & 0.98 & 1.22 \\
\hline 2 & 0.39 & 0.21 & 0.15 \\
\hline 3 & 0.44 & 0.27 & 0.25 \\
\hline 4 & 0.76 & 0.71 & 0.87 \\
\hline 5 & $<0.01$ & 0.36 & 0.28 \\
\hline 6 & 0.04 & 0.03 & 0.02 \\
\hline 7 & 0.15 & 0.11 & 0.11 \\
\hline 8 & 0.36 & 0.37 & 0.18 \\
\hline 9 & 0.21 & 1.06 & 0.85 \\
\hline 10 & 0.16 & 0.97 & 1.25 \\
\hline 11 & 0.51 & 0.53 & 0.62 \\
\hline 12 & 0.08 & 0.65 & 0.39 \\
\hline 13 & 0.22 & 0.24 & 0.26 \\
\hline 14 & $<0.01$ & $<0.01$ & $<0.01$ \\
\hline 15 & 0.08 & 0.15 & 0.08 \\
\hline 16 & 0.51 & 0.38 & 0.30 \\
\hline 17 & $<0.01$ & 2.23 & 2.17 \\
\hline 18 & $<0.01$ & 0.14 & 0.09 \\
\hline
\end{tabular}

Only modest elevation without the rise and fall characteristic of acute coronary syndromes can be seen. Although patients 9, 10 and 17 appear to have significant up-trending in troponins, follow-up echocardiograms in all these patients showed normalisation of left ventricular function function and/or resolution of wall motion abnormalities.

TnT = troponin $T$.

without any significant upward or downward trend, in contrast to the classical peaking noted in acute coronary syndromes. This troponin trend further supported the absence of a plaque rupture event that may have initiated the decrease in LV function or wall motion abnormalities noted on echocardiograms.

The most common echocardiographic wall motion abnormalities noted were isolated mid ventricular hypokinesis and apical ballooning, occurring in $39 \%$ and $33 \%$ of the patients, respectively (Table $4{ }^{3}$ Figure 2). Other echocardiographic patterns were not as frequent. Only limited follow-up data was available in four patients because of in-hospital mortality. In two of these patients, follow-up echocardiograms showed normalisation of LV function with no residual wall motion abnormalities and cardiac catheterisation showed normal coronaries in one patient. One patient had a follow-up echocardiogram within 2 days of the first study which did not show improvement in cardiac function yet. Echocardiographic follow-up data in all other patients showed resolution of wall motion abnormalities, confirming the diagnosis of SSC as per our criteria. Not surprisingly, cardiac catheterisation data was only available in $28 \%(n=5)$ patients, and showed non-obstructive CAD or normal coronaries.

The average follow-up duration in our patient series was 7 months. Six (33\%) patients died over the course of follow-up. Out of these patients, five (28\%) died within 30 days and four (22\%) died during hospitalisation. This underscores the significantly high mortality burden faced by these patients, likely due to underlying critical illnesses.

\section{Discussion}

A significant proportion of critically ill patients develop ECG, troponin, or echocardiographic abnormalities suggestive of SC during the course of their hospitalisation. Many of these patients meet the current definition of 
Table 4: The key clinical, morphological, imaging and outcome differences between primary and secondary stress cardiomyopathy

\begin{tabular}{|c|c|c|}
\hline & $\begin{array}{l}\text { Primary stress cardiomyopathy (data from the } \\
\text { InterTAK Registry })^{3}(n=1,750)\end{array}$ & Secondary stress cardiomyopathy $(\mathrm{n}=18)$ \\
\hline Age range, years & $60-75$ & 21-86 (average 61) \\
\hline Male, n (\%) & $179(10)$ & $5(28)$ \\
\hline Female, n (\%) & $1,571(90)$ & $13(72)$ \\
\hline Angina, n (\%) & $1,229(76)^{*}$ & $0(0)$ \\
\hline Clinical picture & $\begin{array}{l}\text { Acute angina, dyspnoea, ischaemic } \\
\text { ECG changes - similar to acute coronary syndrome }\end{array}$ & $\begin{array}{l}\text { Non-cardiac critical illness, neuro-psychiatric } \\
\text { symptoms, non-specific symptoms }\end{array}$ \\
\hline $\begin{array}{l}\text { Echocardiographic regional wall motion abnormality } \\
\text { patterns, } n \text { (\%) }\end{array}$ & $\begin{array}{l}\text { Apical ballooning 1,430 (82) } \\
\text { Mid ventricular } 255(15) \\
\text { Basal variant } 39(2) \\
\text { Focal variant } 26(2) \\
\text { Global hypokinesia } 0(0)\end{array}$ & $\begin{array}{l}\text { Apical ballooning } 6 \text { (33) } \\
\text { Mid ventricular } 7 \text { (39) } \\
\text { Basal variant } 2 \text { (11) } \\
\text { Focal variant } 1 \text { (6) } \\
\text { Bi-ventricular/global } 2 \text { (11) }\end{array}$ \\
\hline Cardiac catheterisation, n (\%) & $1,750(100)$ & $5(28)$ \\
\hline Shock, n (\%) & $170(10)$ & $8(44)$ \\
\hline Hospital mortality, n (\%) & $72(4)$ & $4(22)$ \\
\hline
\end{tabular}

Data from InterTAK registry is used for primary stress cardiomyopathy. ${ }^{3}$ All percentages given have been rounded to whole numbers.

*Per the INTERTAK registry data, this was calculated as 1,229/1,619.3

Figure 2: Morphologic variants in secondary stress cardiomyopathy in our series

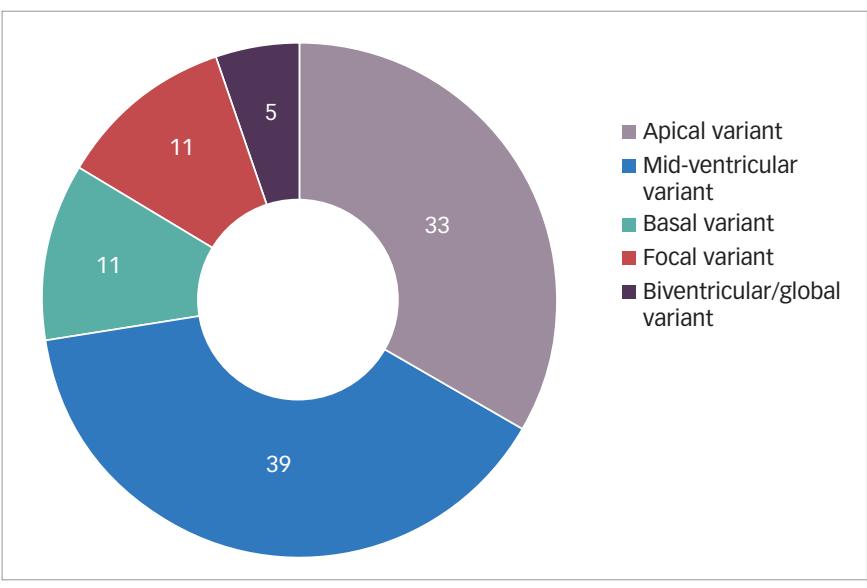

Morphologic variants are expressed in percentages (\%). $n=18$.

$\mathrm{SSC}^{2}$ since they present to the medical setting without any major cardiac symptoms and develop abnormalities suggestive of SC during the course of illness. Given the presence of concomitant comorbidities and other acute medical issues that take precedence, many of these patients are not ideal candidates for cardiac catheterisation to exclude obstructive CAD or plaque rupture. Elevations in serum troponin in these patients may be related to a variety of causes like sepsis, shock, hypoxia, respiratory failure, intracranial haemorrhage, stroke and inotrope use, and appear to be an independent predictor of poor prognosis in these patients with sepsis. Even when such patients are diagnosed with SC based on current Mayo criteria, ${ }^{5}$ clinical course and outcomes should not be presumed to be similar to those predicted by currently available data. ${ }^{3}$ The characterisation of the clinical presentation and outcomes of SSC are therefore very relevant to clinicians taking care of these critically ill patients.

Our study highlights several features of SSC which appear to be different from the widely recognised takotsubo or pSC. It affects a higher proportion of younger patients and males. These patients commonly present without angina. Atypical patterns of wall motion abnormalities on echocardiography, especially the mid-ventricular variant, appear more common in SSC. Other forms, including basal hypokinesis and biventricular/global hypokinesis, also seem to be more common in SSC, whereas apical ballooning accounts for over $80 \%$ of pSC cases. ${ }^{3}$ It is also important to note that patients with SSC have a poor outcome with a 30 -day mortality rate of $28 \%$ in our series. pSC could be complicated by hypotension, cardiogenic shock, dynamic outflow obstruction, malignant arrhythmias, or cardiopulmonary arrest with a 30-day mortality of $4 \%{ }^{3}$ The much higher mortality in SSC is likely due to underlying critical illness.

Overlap of several clinical features makes the differentiation of pSC and SSC difficult in some situations. As noted in our series, while some patients might have had a significant mental stress leading up to attempting suicide or from motor vehicle accident, by the time echocardiographic wall motion abnormalities were noted, these patients were already in the intensive care unit. Often, it is challenging to identify the primary problem. Some patients with cardiopulmonary arrest feature in this series because cardiac dysfunction was noticed in the intensive care unit post-resuscitation. However, there is no way to completely exclude the possibility of SC being the primary issue, leading to heart failure or arrhythmia that degenerates into cardiopulmonary arrest. History of angina just prior to the arrest, absence of underlying cardiac comorbidities or other significant metabolic derangements may suggest pSC in some of these instances.

While apical ballooning with anterior and apical wall motion abnormality may be challenging to differentiate from left anterior descending artery disease and acute coronary syndrome, the basal, mid-ventricular and bi-ventricular variants do not conform to a single coronary territory. This might play a crucial role in the early recognition of SC noninvasively, especially in those who do not present to medical attention for acute cardiac problems. For patients who have prolonged hospitalisation, follow-up echocardiograms showing resolution of regional wall motion abnormalities may be a reliable indicator of SSC. As noted in this small series, coronary angiograms in such patients typically showed absence of obstructive CAD or plaque rupture. 
Although this study suggests several important differences in the presentation of SSC and PSC, large scale data are needed to confirm these findings and further characterise the entity of SSC. There is some room for bias since this is a single-centre experience with patients chosen by a single physician, although we have been publishing diagnostic methods on SSC over the last decade.9.10 Given the complicated comorbidities typically associated with critically ill patients, coronary angiography should be considered as an additional diagnostic tool in a selected group of patients in whom the benefits of angiography outweigh the risks. It is also important to look at the trend of serum troponins during the course of illness, which may identify patients with type I myocardial infarction. This may avoid misclassification of patients who have resolution of wall motion abnormalities following revascularisation, or in the setting of myocardial stunning. Myocarditis should be considered in the differential in the appropriate clinical scenario and magnetic resonance imaging can be considered for additional non-invasive evaluation in this setting.

\section{Conclusion}

Our consecutive SSC patient series illustrates the significant differences in clinical presentation, morphological variants, age distribution and clinical outcomes in these patients. It also highlights the importance of serial echocardiography for diagnosis and management, since invasive catheterisation is neither safe nor feasible for many such patients. sSC carries much a higher mortality risk. High clinical suspicion and non-invasive diagnosis may help optimise outcomes in these challenging patients. Serial data on larger patient populations with SSC are needed to definitively establish the clinical characteristics of this entity..
1. Sato $\mathrm{H}$, Uchida T, Dote $\mathrm{K}$, et al. Tako-tsubo-like ventricular dysfunction due to multivessel coronary spasm. In: Kodama K Haze, K, Hori M., ed. Clinical Aspect of Myocardial Injury: From Haze, K, Hori M., ed. Clinical Aspect of Myocardial Injury: From
Ischemia to Heart Failure. Tokyo: Kagakuhyoronsha Publishing; Ischemia to

2. Lyon AR, Bossone E, Schneider B, et al. Current state of knowledge on takotsubo syndrome: a Position Statement from the Taskforce on Takotsubo Syndrome of the Heart Failure Association of the European Society of Cardiology. Eur J Heart Fail. 2016;18:8-27.

3. Templin C, Ghadri JR, Diekmann J, et al. Clinical features and outcomes of takotsubo (stress) cardiomyopathy. N Engl J Med. 2015;373:929-38.
4. Bybee KA, Kara T, Prasad A, et al. Systematic review: transient left ventricular apical ballooning: a syndrome that mimics ST-segment elevation myocardial infarction. Ann Intern Med. 2004; 141:858-65.

5. Prasad A, Lerman A, Rihal CS. Apical ballooning syndrome (Tako-Tsubo or stress cardiomyopathy): A mimic of acute myocardial infarction. Am Heart J. 2008;155:408-17.

6. Medina de Chazal H, Del Buono MG, Keyser-Marcus L, et al. Stress cardiomyopathy diagnosis and treatment: JACC State-of-the-Art Review. J Am Coll Cardiol. 2018;72:1955-71.

7. Ghadri JR, Cammann VL, Jurisic S, et al. A novel clinical score (InterTAK Diagnostic Score) to differentiate takotsubo syndrome from acute coronary syndrome: results from the International Takotsubo Registry. Eur I Heart Fail. 2017;19:1036-42.

8. Nunez-Gil IJ, Almendro-Delia M, Andres M, et al. Secondary forms of takotsubo cardiomyopathy: A whole different prognosis. Eur Heart J Acute Cardiovasc Care. 2016;5:308-16

9. Chockalingam A, Xie GY, Dellsperger KC. Echocardiography in stress cardiomyopathy and acute LVOT obstruction. Int I Cardiovasc Imaging. 2010;26:527-35.

10. Chockalingam A. Stress cardiomyopathy of the critically ill: Spectrum of secondary, global, probable and subclinical forms. Indian Heart J. 2018;70:177-84. 